\title{
BIOECONOMY: SHAPING THE TRANSITION TO A SUSTAINABLE, BIOBASED ECONOMY, edited by Iris Lewandowski (Springer, 2018)
}

\author{
MERVE BURÇAK KETENE ${ }^{l}$
}

Today's increasing demands for food, feed, products and energy, in conjunction with the security concerns brought about by a growing global population and anthropogenically induced climate change, are making the need for a sustainable system more pressing than ever. In this context, to reflect the need for a comprehensive societal transformation, the bio-based or knowledge-based economy is targeting a reduction in the present dependency on fossil fuels along with other forms of finite resources through their gradual replacement by renewables. However, while the objectives of such a major system change are mainly agreed upon, the pathway to achieving them is still a subject of dispute. Having the purpose of engaging in the debate, and attempting to lay the keystones for shaping the future, this book, Bioeconomy, explores and conveys the advantages of a sustainable, innovative and knowledgebased bioeconomy from an inter- and transdisciplinary perspective. Its aim is to advance the existing knowledge and to pioneer national as much as international cooperation in the field. The book's editor, Iris Lewandowski, is known for her involvement in national and EU-funded bioeconomy research projects. One of these is called "Accelerating the transition towards a BioBased Economy via Education - ABBEE" which was initiated as of September 1, 2018 with the objective of creating new educational materials for students and professionals that incorporate the key elements of a biobased economy. Currently, the author is affiliated with the University of Hohenheim, where she is the Chief Bioeconomy Officer, and is also responsible for an international master's program on Bioeconomy. Taking these facts into consideration, and framing them in the form of a textbook, this joint venture of the University of Hohenheim's educators and students has resulted in Bioeconomy, which

\footnotetext{
1 Merve Burçak Ketene is a Ph.D. student at the Doctoral School of Sociology, Corvinus University of Budapest; e-mail: merve.ketene@stud.uni-corvinus.hu
} 
combines principles from agriculture, biotechnology and macroeconomics, while presenting the complexities of the field holistically.

The book has three major parts that contain 12 chapters in total, each investigating a different aspect of the concept of bioeconomy. Right from the very start - the first chapter of Part I - the authors present the contextual background of the topic and introduce some key terminological notions such as the difference between fossil energy reserves and resources, greenhouse gases (GHG), climate change, biobased resources, planetary boundaries and limits to natural resources, food security and sustainable bioeconomy. Among these, the sub-chapter entitled "Planetary Boundaries and Limitation of Natural Resources" is of particular value since it underlines the fact that climate change is only one of the nine planetary boundaries which are crucial in terms of the carrying capacity of the Earth - in other words, crucial for humanity's continuing existence. While "climate change and land-system change processes are already beyond the safe operating space," as reported by the authors, there are two specific categories of planetary boundaries that indicate much greater risk. The first is biosphere integrity, particularly in terms of genetic diversity; and the other is the biogeochemical flows of nitrogen and phosphorus to the biosphere and oceans "as a result of various industrial and agricultural processes." In this regard, because the need for food is not likely to decrease, the authors stress the benefits of the intensification of sustainable agriculture. Two terms should be clarified here: first of all, sustainability is amongst the major goals of the bioeconomy. Being directly dependent on the availability of natural resources, the reasonable and efficient utilization of these and therefore their sustainability is prioritized within this system. Second, agricultural intensification is a process that is based on the principle of producing more without additional land. This, of course, makes innovation an integral part of production processes. Remarkably, the authors highlight that $70 \%$ of total factor productivity in agriculture is actually created by innovation, whereas the expansion of land area accounts for a share of only $12 \%$. Added to this is increasing concern about food security resulting from global population growth. When these factors, taken together with the fact that meat production requires more land than the production of crops, and the authors' observation concerning an upward trend in meat consumption, particularly within emerging economies, the need for a sustainable solution becomes urgent as regards to such global challenges. Recall that the bioeconomy itself is highly dependent on the availability of natural resources, thus it is also subject to the need for innovative sustainability. Thereof, the authors lay emphasis on the following: 
"In a sustainable bioeconomy, the use of biobased resources should be optimized with regard to two main criteria: First, the demand for highquality food for the world's population should be satisfied. Second, the remaining biobased resources should ideally be allocated with regard to the maximal ecological, social and economic benefit." (p. 14)

This holistic resource allocation principle, along with a call for a knowledgebased transition to a system that is both sustainable and innovative, and which perceives the willingness to participate of consumers and producers as indispensable, are the distinguishing elements of the bioeconomy. For all that, the presented discussion lacks explicit attention to the areas of waste management, water utilization, and issues with logistical concerns regarding bio-based production. It is true that the bioeconomy has the potential to revitalize rural areas through creating new job opportunities, particularly in places where fossil resources are scarce but biobased ones are abundant. However, "the transportability of biomass is often limited due to its low density and susceptibility to decaying" - which puts the whole process in jeopardy of being bounded and having only regional applicability at most. Ways of overcoming such barriers, on the other hand, are not thoroughly explored, apart from the flatly underlined requirement for strong regional cooperation. Having made these observations, the authors then continue in Part I with explanations of the origin and evolution of the bioeconomy, wherein they identify the biotechnology innovation perspective and the resource substitution perspective as two dimensions of this concept. Thankfully, they do not neglect to mention the existing criticisms of bioeconomy; namely, the "fundamental critique" that refers to the discourse as dominantly siding with "neoliberal ideology," and the "greenwashing critique," which acknowledges the potential of the bioeconomy while trying to "ensure that the label 'bio' is not misused to portray an essentially non-sustainable economic system as environmentally friendly." Later on, the concept of circular economy and its links with the bioeconomy are explored; moreover, the "Diamond Model" of gaining competitive advantage through bioeconomy is introduced as a country-dependent strategy. The last chapter in Part I addresses the "wicked" problems of bioeconomy that are complex, in addition to being economically, environmentally and socially interwoven. These problems require researchers to synthesize knowledge from various sources by putting inter- and transdisciplinary research into practice, while being cautious about the potential impacts of their individual norms and values on research outcomes in such participatory cooperation processes.

Part II complements Part I in terms of knowledge building. As the authors regularly emphasize during the previous chapters, and stress further in the last 
chapter of this book called The Bioeconomist, professionals working in the field of bioeconomy must possess certain skills and a sophisticated understanding of almost all areas of bioeconomy to be able to design and conduct transdisciplinary research. Based on this view, the second part of Bioeconomy offers an in-depth exploration of biobased resources. Although the chapters are elaborative, particularly in the areas concerning primary production, regarding bioenergy there is disappointingly limited coverage. The latter is briefly addressed as an important sector of the bioeconomy that "requires subsidies to be economically viable" - while further discussion of these subsidies is missing. Also, since this topic is strongly connected to the food vs. fuel debate, as logic dictates, the issue of energy security should not be an area of neglect. Forging ahead with the upsides, several important concepts emerge from the chapters of Part II. One of those is biobased value chains that "describe[...] the full range of activities which are required to bring a product or service from conception, through the different phases of production [...], delivery to final consumers, and final disposal after use." These are considerable assets for stakeholders and for innovation purposes, and within the bioeconomy they take the form of a cascade that can be used to maximize "socioeconomic value given the constraint of resource limitation." Thus, cascading biobased value chains can be understood as biomass application management that transforms "linear production processes into circular or closed ones, accordingly reducing the generation of waste." Yet, of course, such activities require close cooperation among many different sectors and a change in mindset, not to mention dealing with questions about how to decide which values should be prioritized to facilitate the arrangement of the sequential utilization of biomass. However, if these issues can be overcome, according to the authors' experience, the development cycles of biobased products could be decreased by half. Another important concept that emerges from the chapters of Part II is the "infant industry" argument put forward for the benefit of fostering the bioeconomy. In a nutshell, the argument is derived from the fact that:

"[A]n increase in the demand and supply of biobased products, and consequently an increase in the amount of crops produced for the biobased market are only possible by a reallocation of land from the production of food to the production of biobased resources. This increase in the demand for land leads to an increase in the price of land, which increases input costs and thus, makes production of biobased products less cost efficient.” (p. 241)

In this framework, if governments introduce temporary support policies for biobased products, which are categorized as biofuels, biochemicals and 
biomaterials, they could compete with existing, already established products. This argument specifically lays emphasis on the market influencing capabilities of bio- and fossil-fuels, since in the case of the latter the environmental (or external) costs, such as their contribution to high carbon emissions, are not reflected in their market price. In addition to government interventions into the market, the authors also focus on the promising potential of private initiatives led by entrepreneurs. Four different types of trends are mentioned that could presumably trigger entrepreneurial opportunities: incongruences and information asymmetries, exogenous shocks, changes in demand, and changes in supply. In any case, it should be mentioned here as a side note that, even if certain initiatives are put forward by various actors from the bioeconomy, there is still the risk of one-way communication that would lock novel innovations and ideas into areas only known about by specific sectors or institutional clusters. This, in turn, could affect the willingness of potential consumers to pay premium prices for bio-based products since this key information has not been transmitted to them. One such issue concerns the existence of hybrid biobased products that are not communicated to the public properly. The authors of Part II provide an exquisite example of this matter called the lotus effect. In this particular application of biological knowledge to the industry, the self-cleaning properties of the lotus plant are technically applied to numerous everyday items such as paints, coatings and roof tiles. Practical and intriguing as this is, unless novel innovations of this kind are duly introduced to consumers, their relevance to markets will continue to be limited. Hence, to lay the ground for triggering the development of bioeconomy, a business model canvas is offered based on what the authors call the lean startup approach. This approach brings forth "an iterative and agile method to develop a startup, (...) [whose] focal question is whether the product or service solves a real problem from real customers and whether a valid business model can be developed." Because the required time span for biobased products is usually longer than conventional ones (the former are sometimes planned far into the future), the proposed lean startup method grounds itself on a minimum viable product that involves testing entrepreneurs' hypotheses in the bioeconomy market as early as possible by helping create a reduced offering to which customers can give immediate feedback. One example that the authors provide is the German startup Betula Manus and their market testing of tree bark, which is a waste product of the paper industry. As is the case here, through the medium of different minimum viable products startups and entrepreneurs can observe potential failures, and identify areas for improvement in their product or service ideas.

In connection with call for the knowledge building required for a sustainable bioeconomy, the final part of the book puts the transition process under the 
microscope. However, such a complex system transition cannot be rolled out only on paper, and it cannot cover just technological or economic issues - it must be preferred and supported by people too. Therefore, to help with understanding and depicting possible transition paths, with all of their uncertainties and variables, researchers rely on certain scenarios and models. To speak bluntly, throughout this entire book on bioeconomy as a new governance system, it is here that the authors have decided to specifically address the role of bioenergy - that is, within the modeling techniques. As one of humanity's biggest sources of dependency, the issue of the constant need for energy and the search for diversified sources, in my opinion, should have received its own chapter. Nevertheless, during the discussion of "techno-economic optimization models" the authors mention bioenergy combustion plants and biorefineries as presenting challenges for model building, and thus needing optimization. Although leaving room for further work, valuable examples are provided throughout the chapter about specific models that particularly focus on the energy sector - such as BeWhere, or BiOLoCaTe mixed linear programming models. Keeping these in mind, the first chapter of Part III in Bioeconomy presents an overview of both scenario and model approaches, while notably distinguishing Integrated Assessment Models (IAMs). By definition, IAMs "describe and assess the interactions between human activities and (global) environmental processes. They include descriptions of socio-economic systems as well as environmental systems and the interactions between the two." These characteristics of IAMs do indicate a relatively more advantageous approach compared to other topicspecific model options, given their holistic nature. Furthermore, IAMs are claimed to have the potential to assess the long-term contributions and impacts of the bioenergy segment of bioeconomy by integrating various different systems into one model - an example of this, as highlighted by the authors, is TIMES PanEU, a multiregional model of the European energy system. The scope of this model includes all sectors of energy supply and demand on the national level; however, when coupled with different sectoral models through IAMs it could become a single part of a much wider system analysis. At the same time, the authors also warn against overestimating the power of IAMs or any other kinds of models to predict the future. They can assist with revealing possible transition paths, yet the actual transformation depends on people and their willingness to change. In explicitly emphasizing the importance of societal and governmental involvement for the transition to a sustainable bioeconomy, the next chapters in Part III analyze the concept of sustainability, the causes of market failure in the environmental sector, social welfare-maximizing functions of government, the transformation of production systems, the neo-Schumpeterian approach, and of course, the bioeconomist as a collaboration catalyst. Taken together, 
all of the arguments that are presented signify the importance of knowledgebased, sustainable production and consumption patterns, in which the conscious involvement of every person indeed makes a difference.

The challenges and the issues of the twenty-first century are overwhelmingly "wicked." They are wicked in the sense of being complex, and undeniably global. They concern the accelerating deterioration of the human environment, as much as of natural resources, and the resonance of these for socio-economic systems. They involve each and every one of us, yet they are also personal. Thus, they do not require simple answers, but effective solutions. Solutions that we can all be on board with, that can be used to sustainably meet today's needs while preserving the same chances for future generations. And this is precisely what Bioeconomy: Shaping the Transition to a Sustainable, Biobased Economy sets its sight on. It explores a variety of perspectives within the field, from biological to economic, presents the current state of existing research, along with the knowledge, and offers pathways for change. The book, throughout its 355 pages, does what it promises to do, introducing various aspects and elements of the bioeconomy while providing concrete instructions for making the desired transition to a sustainable, biobased economy. It is a comprehensive book that, on occasion, will challenge the reader to grasp all of the details, case studies, economic calculations and topic-specific terminology. However, such a challenge emerges from the aim of contemplating Bioeconomy as a text book rather than a light read. On this note, certain areas of further attention, some of which are mentioned earlier in this review, should not be overlooked. Briefly addressing a few, first of all, there is an absolute lack of discussion about energy options - be they renewables, biofuels or nuclear power. A short chapter on biofuel types and biobased resource processing, without a presentation of related energy charts, cannot be sufficient for educating the future's experts about crucial aspects of the bioeconomy. Also, given the fact that all technologies have their own challenges that must be resolved, national self-sufficiency and energy security concerns would surely benefit from resource diversification techniques that can secure supplies - supplies that, among many other uses, will also be needed at every stage in the cascading use of biomass. In this context, energy generation options associated with zero $\mathrm{CO}_{2}$ production, such as nuclear power or renewables, should not be disregarded in any discussion of non-biological alternatives to biomass sources. Second, water utilization and especially pollution needs much more attention since it is an active issue in biorefinery operations. Likewise, the drawbacks of biomass use are not thoroughly explored. Questions remain regarding the risk of reducing the overall carbon storage capacity of forests through their over-exploitative use, the difference between intact and selectively logged forests in terms of carbon sequestering, and pollutant-related issues with 
biofuels and biomass which, much like with fossil fuels, occur when they are not combusted properly. These cases should be addressed and take their place on the agenda of creating solutions for a sustainable, low-carbon future. And finally, although there is substantial discourse about the transition process, the book does not provide an approximate timeframe for it. This is of considerable importance, because, depending on whether such a system change occurs tomorrow, in five years' time, or in ten, there will be a drastic difference in cost - both in terms of the environment, and the socio-economic systems. Nevertheless, these considerations are presented to support the transition from a fossil-based economy to a biobased one; to identify the scope of this participative governance system and work on expanding it through proper management and constant technological development; but most of all, to reduce our current dependency on fossil fuels, perhaps for good. From this perspective, the authors of this book make Bioeconomy a must-read for experts and students, as well as for those interested in the topic, who are wondering about how to become more consciously involved in our century's increasingly interconnected challenges. After all the analyses, models and discussions about the role of the bioeconomy in "shaping the transition to a sustainable" future, this is the underlying purpose of the book in terms of its readers: Obtain the knowledge, and get involved. 